\title{
Desempenho produtivo e econômico do confinamento de bovinos zebuínos alimentados com três dietas de alto concentrado
}

\author{
[Economic performance of zebu cattle under feedlot fed with three high concentrate diets] \\ R.A. Mandarino ${ }^{1}$, F.A. Barbosa ${ }^{2}$, S.L.S. Cabral Filho ${ }^{1}$, C.F. Lobo ${ }^{1}$, I.S. Silva ${ }^{1}$, \\ R.V. Oliveira ${ }^{1}$, J.M.S. Diogo ${ }^{1}$, R. Guimarães Júnior ${ }^{3}$ \\ ${ }^{1}$ Universidade de Brasília - Faculdade de Agronomia e Medicina Veterinária - Brasília, DF \\ ${ }^{2}$ Universidade Federal de Minas Gerais - Escola de Veterinária - Belo Horizonte, MG \\ ${ }^{3}$ Empresa Brasileira de Pesquisa Agropecuária - Embrapa - Planaltina, DF
}

\begin{abstract}
RESUMO
Estudaram-se o desempenho produtivo e a viabilidade econômica no confinamento de 24 bovinos, inteiros, com idade média de 23 meses, sendo 12 Nelore (NEL) e 12 F1 Nelore x Brahman (NBR), divididos em três dietas: SIL - silagem de milho e ração concentrada; PEL - exclusiva de pellets; GRN milho grão inteiro e pellets. $\mathrm{O}$ experimento foi realizado em um delineamento inteiramente ao acaso, em esquema fatorial $2 \times 3$, com quatro repetições. Os animais NBR apresentaram resultados semelhantes aos da raça NEL quanto aos desempenhos alimentar e econômico, para todas as variáveis analisadas durante o confinamento. Entre as três dietas, a dieta PEL, apesar de não ser a de maior custo em $\mathrm{R} \$ / \mathrm{kg}$, obteve o menor desempenho, com maior COT/kg, menor RT e menores margens líquidas. A dieta SIL apresentou os menores COT $/ \mathrm{kg}$ e custo $/ \mathrm{kg}$ de carcaça, com maiores receitas e margens líquidas. A dieta GRN obteve resultados econômicos semelhantes à SIL, porém obteve menor GMD em relação à SIL, além de ter o custo de dieta $(\mathrm{R} \$ / \mathrm{kg})$ mais oneroso em relação aos três tratamentos. Todas as dietas foram economicamente viáveis ao final do experimento, com $\mathrm{ML} / \mathrm{Kg}$ de carcaça médios de 2,12, 0,35 e 1,09 para SIL, PEL e GRN, respectivamente, no entanto a dieta SIL foi a de melhor desempenho produtivo e econômico.
\end{abstract}

Palavras-chave: Brahman, custos, Nelore, grão inteiro

\begin{abstract}
The production performance and economic viability of a feedlot of 24 cattle, with an average age of 23 months, 12 Nellore (NEL) and 12 F1 Nellore x Brahman (NBR), divided into three diets: SIL - corn silage and concentrate diet; PEL - exclusive of pellets; GRN - corn and whole grain pellets were studied. The experiment was conducted in a completely randomized scheme in a $2 x 3$ factorial with four replications. The NBR animals showed similar results to the NEL in performance and economic viability for all variables analyzed. Among the diets, the PEL diet, although not the highest diet cost in R\$/kg, had the lowest performance with higher TOC/kg, lower TR and lower net margins. The SIL diet had the lowest TOC/kg and cost $/ \mathrm{kg}$ of carcass, with higher revenues and net margins. The economic results obtained for the GRN diet were similar to SIL, but had lower ADG compared to SIL and highest diet cost $(R \$ / \mathrm{kg})$ among all. The diets were economically viable at the end of the trial, with average net margin/ $\mathrm{kg}$ carcass of 2.12, 0.35 and 1.09 for SIL, FEP and GRN, respectively; however the SIL diet presented better productive and economical performance.
\end{abstract}

Keywords: Brahman, costs, Nellore, whole grain

Recebido em 5 de março de 2012

Aceito em 6 de março de 2013

E-mail: raphael@mandarino.com.br 


\section{INTRODUÇÃO}

Com o conhecimento dos custos de produção, o agricultor ou o técnico pode avaliar a atividade econômica e, por meio desta análise, aprender em detalhes a usar alguns fatores econômicos de produção como: terra, capital, trabalho e capacidade gerencial (Lopes e Magalhães, 2005).

Segundo Bungenstab (2001), somente a adoção de tecnologias não basta para garantir a lucratividade ao setor de pecuária de corte, especialmente no sistema intensivo. $\mathrm{O}$ gerenciamento é fundamental e o controle de custos é a ferramenta para alcançar um bom gerenciamento. A nova ordem econômica vem exigindo do produtor rural uma visão mais profissional da administração de seus negócios, demonstrando que a agropecuária apresenta o mesmo nível de complexidade, importância e dinâmica dos demais setores da economia, como indústria, comércio e serviços (Lopes e Carvalho, 2002).

Nos cruzamentos entre raças zebuínas ou entre raças taurinas, a heterose não fica tão evidente (Ribeiro et al., 2008). Porém, animais F1 Nelore $\mathrm{x}$ Brahman mostraram superioridade de peso à desmama e ao abate, e maior rendimento de carcaça (Cundiff et al., 1993; Ribeiro et al., 2008), frutos de efeitos genéticos aditivos e da heterose.

Devido ao fato de apenas uma pequena parte da indústria brasileira trabalhar com animais provenientes de confinamentos e do alto número de incidência de cruzamentos em rebanhos comerciais, a necessidade de estudos no sentido de avaliar a capacidade produtiva e a viabilidade econômica é importante para planejamentos futuros. Sendo assim, o objetivo deste trabalho foi analisar o desempenho produtivo e a viabilidade econômica do confinamento de animais Nelore e F1 Nelore x Brahman sob três regimes alimentares.

\section{MATERIAL E MÉTODOS}

O estudo foi conduzido na fazenda da Universidade de Brasília - UnB, Brasil, localizada no Distrito Federal (1556'12.66" de latitude sul e $47^{\circ} 55^{\prime} 12.55^{\prime \prime}$ de longitude oeste, no Distrito Federal, altitude de cerca de $1.000 \mathrm{~m}$ ), com início em 21 de agosto e término em 25 de novembro 2009, em um período experimental de 96 dias.
Foram utilizados 24 bovinos, 12 Nelore (NEL) e 12 F1 Nelore x Brahman (NBR), com idade média de 23 meses e do mesmo rebanho comercial. O período de adaptação foi de 21 dias, e os animais foram alimentados com uma dieta ad libitum, composta de volumoso de $50 \%$ (silagem de milho) e $50 \%$ de concentrado, sendo aumentado gradualmente o nível de concentrado para a proporção de concentrado:volumoso de 75:25 (base na MS). Nas dietas exclusivas de concentrado, foi ofertada silagem de milho, inicialmente com 50\% (base MS), com redução gradativa até o dia inicial do experimento, quando o nível de volumoso foi zero e o de concentrado $100 \%$.

$\mathrm{Na}$ Tab. 1, são mostradas as composições das dietas e a proporção de alimentos utilizadas nas formulações.

Cada grupo genético foi dividido em três grupos diferentes conforme a dieta, com quatro animais em cada: SIL - silagem de milho e concentrado (grão de milho, farelo de soja, casca de soja, ureia e suplemento mineral) na proporção de 25:75 volumoso:concentrado (base na MS); PEL - dieta exclusiva de concentrado em pellets; GRN - dieta com $85 \%$ de milho grão inteiro e $15 \%$ de concentrado em pellets. As dietas foram fornecidas ad libitum e divididas em três refeições diárias (8, 11 e 15 horas). Os animais tinham ingestão ad libitum de água. A quantidade de alimento fornecido foi pesada diariamente e ajustada de forma a sempre permitir 5 a $10 \%$ de sobras. As sobras foram pesadas e registradas diariamente antes da primeira refeição do dia

O consumo individual de matéria seca (CIMS) foi determinado utilizando-se óxido de cromo $\left(\mathrm{Cr}_{2} \mathrm{O}_{3}\right)$ como indicador externo, dividido em dois períodos, início no dia 30 e no dia 60 , durante o confinamento. $\mathrm{O}$ óxido de cromo foi fornecido em papel embrulhado e introduzido via oral durante sete dias consecutivos, em uma dose diária de 10 gramas às oito horas. No quarto dia após a administração, foram coletadas as fezes diretamente do reto do animal. Amostras de fezes foram analisadas para teor de cromo de acordo com a técnica de Williams et al. (1962), utilizando-se espectrofotômetro de absorção atômica no Laboratório de Química Analítica, Departamento de Química, UnB, Brasil. 
Tabela 1. Proporção dos ingredientes e composição nutricional das dietas (\% na matéria seca)

\begin{tabular}{lccc}
\hline \multicolumn{1}{c}{ Ingredientes (\%) } & SIL & PEL & GRN \\
\hline Silagem de milho & 25 & - & - \\
Casca de soja & 35 & - & - \\
Ureia & 0,98 & - & - \\
Milho & 31,89 & - & 85 \\
Farelo de algodão & 5,82 & - & - \\
Suplemento peletizado para dieta alto grão & - & - & 15 \\
Suplemento peletizado & - & 100 & - \\
Mistura mineral & 1,2 & - & - \\
Calcário & 0,11 & - & - \\
\hline & 100 & 100 & 100 \\
\hline Matéria seca (\%) & 63,5 & 86,79 & 85,25 \\
Proteína bruta $(\%)$ & 13,41 & 16,52 & 17,6 \\
Extrato etéreo (\%) & 4,92 & 3,26 & 3,53 \\
Nutrientes digestiveis totais $\dagger(\%)$ & 85,43 & 89,19 & 88,48 \\
Fibra em detergente neutro $(\%)$ & 38,88 & 43,36 & 13,66 \\
Fibra em detergente ácido $(\%)$ & 21,11 & 26,61 & 8,83 \\
ED (Mcal/kg MS) ${ }^{*}$ & 3,77 & 3,93 & 3,90 \\
EM (Mcal/kg MS) ${ }^{*}$ & 3.08 & 3.22 & 3.20 \\
ELm (Mcal/kg MS) $*$ & 1.49 & 1.51 & 1.51 \\
ELg (Mcal/kg MS) ${ }^{*}$ & 1.44 & 1.53 & 1.51 \\
Matéria mineral (\%) & 2,79 & 4,87 & 5,41 \\
Digestibilidade in vitro da matéria seca $(\%)$ & 83,90 & 87,44 & 86,77 \\
\hline
\end{tabular}

$\uparrow$ Cappele et al. (2001); NDT= -3,84+1,064 DIVMS.

$*$ NRC (Nutrient..., 1996). ED (Mcal/kg MS) = 0,04409*\%NDT; EM= 0,82*ED; ELg $(\mathrm{Mcal} / \mathrm{kgMS})=-1,65+1,42$ $\mathrm{EM}-0,174 \mathrm{EM}^{2}+0,0122 \mathrm{EM}^{3}$.

SIL - silagem de milho e concentrado (grão de milho, farelo de soja, casca de soja, ureia e suplemento mineral) na proporção de 25:75 volumoso:concentrado (base na MS); PEL - dieta exclusiva de concentrado em pellets; GRN dieta com $85 \%$ de milho grão inteiro e $15 \%$ de concentrado em pellets.

A produção fecal (PF) e o CIMS foram calculados de acordo com Smith e Reid (1955). Para obter a digestibilidade in vitro da matéria seca (DIVMS), as dietas foram submetidas ao procedimento de digestibilidade in vitro de dois estágios, proposta por Tilley e Terry (1963), no Laboratório de Nutrição de Ruminantes da Embrapa Cerrados, localizada em Brasília/DF. O GMD foi calculado como a diferença entre o peso vivo final (PVF) e o peso vivo inicial (PVI), dividido pelo número total de dias. A eficiência alimentar (EF) foi calculada como o quociente entre o GMD e o CIMS. O rendimento de carcaça quente (RCQ) de cada animal foi calculado pela seguinte fórmula: $\mathrm{RCQ}=$ peso da carcaça quente/PVF x 100.

Todos os animais foram abatidos 24 horas após o último dia do experimento. Os animais foram pesados de manhã, antes de serem enviados para o frigorífico. No frigorífico, eles ficaram em um jejum de sólidos por 24h, com ingestão ad libitum de água. Todos os animais foram sacrificados por concussão cerebral, seguida por secção da veia jugular, de acordo com o MAPA (Brasil, 2000).

Os dados econômicos foram coletados por meio de fichas de controle e analisados utilizando-se planilhas (Microsoft Excel). O custo operacional total (COT) foi calculado pela somatória do custo operacional variável (COV) de cada dieta, compostos pelos custos com alimentação (SILR\$ 369,90; PEL - R\$ 309,40 e GRN - R\$ $333,70)$, obtidos pelo somatório dos gastos com os alimentos oferecidos durante o confinamento e dos custos operacionais fixos (COF), sendo usado o valor de $\mathrm{R} \$ 18,77 /$ bovino com a mão de obra e R\$ 6,99/bovino com a depreciação dos bens. A receita total por $\mathrm{kg}$ de carcaça foi 
obtida pela quantidade de carcaça produzida multiplicada por $\mathrm{R} \$ 5,00$ (valor recebido pela venda dos animais por $\mathrm{kg}$ de carcaça). A margem líquida por $\mathrm{kg}$ de carcaça foi obtida pela diferença entre a receita total $/ \mathrm{kg}$ de carcaça e o $\mathrm{COT} / \mathrm{kg}$ de carcaça. Os indicadores econômicos foram calculados pelo valor de mercado de cada insumo na época e com base nos indicadores utilizados por Corrêa et al. (2000), Reis (2002) e Nogueira (2004).

As dietas foram analisadas no Laboratório de Nutrição Animal, FAL/UnB, de acordo com a metodologia de Kjeldahl para proteína bruta (PB), com as recomendações da AOAC (Association..., 1990). Também foram analisadas para matéria seca (MS), extrato etéreo (EE), cinzas e fibra em detergente ácido (FDA). Analisadas ainda para fibra em detergente neutro (FDN) de acordo com Van Soest et al. (1991). Os nutrientes digestíveis totais (NDT) foram calculados com base na digestibilidade in vitro da matéria seca (DIVMS) pela equação proposta por Cappele et al. (2001).

O experimento foi conduzido em um esquema de delineamento inteiramente ao acaso, em um fatorial 2x3 (dois genótipos e três dietas), dividido da seguinte forma: NELSIL, NELPEL, NELGRN, NBRSIL, NBRPEL e NBRGRN. Os dados foram analisados adotando-se o seguinte modelo estatístico:

$$
Y_{i j k}=\mu+a_{i}+b_{j}+(a b)_{i j}+P I_{i}+E_{i j k},
$$

em que: $Y_{\mathrm{ijk}}=\mathrm{a}$ variável dependente da observação i do grupo genético, nível de dieta j e repetição $\mathrm{k} ; \mu=$ uma constante, $\mathrm{a}_{\mathrm{i}}=$ efeito do $\mathrm{i}$ ésimo grau do nível do grupo genético, em que 1 $=$ Nelore e 2 = Nelore $x$ Brahman; $b_{j}=$ efeito do j-ésimo grau do nível da dieta, em que $1=$ SIL 2 $=$ PEL e $3=$ GRN, $(\mathrm{ab})_{\mathrm{ij}}=$ interação entre grupo genético i e dieta $\mathrm{j} ; \mathrm{PI}_{\mathrm{i}}=$ covariável peso inicial do animal $\mathrm{i} ; \mathrm{E}_{\mathrm{ijk}}=$ erro experimental do grupo genético $\mathrm{i}$, da dieta $\mathrm{j}$ e da repetição $\mathrm{k}$.

Todos os dados de desempenho foram analisados usando-se PROC GLM (Statistical..., 2001) com o teste de Duncan, em um nível de significância de $5 \%$. Os dados econômicos foram submetidos à análise de variância, com comparação das médias, a $5 \%$ de probabilidade, pelo teste Tukey, por intermédio do programa estatístico SAS (Statistical..., 2001).

\section{RESULTADOS E DISCUSSÃO}

$\mathrm{O}$ peso vivo final (PVF) diferiu estatisticamente $(\mathrm{P}<0,05)$ entre as dietas avaliadas. É possível observar que SIL proporcionou maior PVF, e PEL proporcionou menor PVF; a dieta GRN foi estatisticamente semelhante à SIL e PEL. Os resultados encontrados para GMD também apresentaram diferenças estatísticas $(\mathrm{P}<0,05)$, sendo a dieta SIL a de maior GMD, seguida por GRN e PEL, todas diferentes entre si $(\mathrm{P}<0,05)$. Para a EF, não houve diferença estatística entre SIL e PEL e entre SIL e GRN $(\mathrm{P}>0,05)$, porém PEL e GRN foram estatisticamente diferentes entre si $(\mathrm{P}<0,05)$. Para as variáveis CIMS, $\mathrm{CMS}^{0,75}$, CPB, CED, PCQ e RCQ, não foram observadas diferenças estatísticas entre si para as diferentes dietas $(\mathrm{P}>0,05)$. Quanto à avaliação entre grupo genético, não houve diferenças estatísticas para nenhuma das características de desempenho avaliadas (P>0,05) (Tab. 2). De acordo com Kippert Júnior et al. (2008), o melhor desempenho observado em animais cruzados se deve ao efeito das heteroses individual e materna, fato que não explica $o$ resultado no presente trabalho em razão da proximidade genética entre Nelore e Brahman.

Benton et al. (2007), ao trabalharem com feno de alfafa em níveis de 4 ou $8 \%$, silagem de milho em níveis de 6 ou $12 \%$ e talos de milho em níveis de 3 ou $6 \%$ na matéria seca (MS) e tratamento controle sem adição de volumoso, relataram valores crescentes para GMD, CMS e $\mathrm{PVF}$ ao se adicionar volumoso na dieta $(\mathrm{P}>0,05)$. Não houve diferença estatística na EF nos diferentes níveis de volumoso $(\mathrm{P}<0,05)$. Resultados semelhantes foram observados no trabalho de Kreikemeier et al. (1990), que, ao trabalharem com níveis de $0,5,10$ ou $15 \%$ de feno de alfafa em dietas de trigo em flocos, observaram que o GMD respondeu quadraticamente, sendo o GMD máximo para $5 \%$ e $10 \%$ de feno de alfafa. 
Desempenho produtivo e econômico...

Tabela 2. Médias e coeficientes de variação de consumo e eficiência de Nelore (NEL) e F1 Nelore x Brahman (NBR), terminados em confinamento, sob três dietas diferentes

\begin{tabular}{|c|c|c|c|c|c|c|c|c|}
\hline & \multicolumn{3}{|c|}{ Dietas } & \multicolumn{2}{|c|}{ Grupo genético } & \multirow{2}{*}{$\mathrm{CV}$} & \multirow{2}{*}{$\begin{array}{c}\text { Valor - } \\
\text { P }\end{array}$} & \multirow{2}{*}{ Erro } \\
\hline & SIL & PEL & GRN & NEL & NBR & & & \\
\hline PVF, kg & $515,43 a$ & $471,36 b$ & $482,14 a b$ & $493,32 \mathrm{a}$ & $488,32 \mathrm{a}$ & 6,55 & 0,22 & 32,17 \\
\hline $\mathrm{GT}, \mathrm{kg}$ & $148,88 \mathrm{a}$ & $91,64 c$ & $120,29 b$ & $131,72 \mathrm{a}$ & $111,40 \mathrm{~b}$ & 14,33 & 0,02 & 17,42 \\
\hline GMD, kg & $1,55 \mathrm{a}$ & $0,95 \mathrm{c}$ & $1,25 b$ & $1,37 \mathrm{a}$ & $1,20 \mathrm{a}$ & 15,51 & 0,01 & 0,18 \\
\hline $\mathrm{EF}, \%$ & $16 a b$ & $10 \mathrm{~b}$ & $17 \mathrm{a}$ & $16 a$ & $14 a$ & 36,2 & 17 & 0,5 \\
\hline CIMS, kg/dia & $9,84 a$ & $9,44 a$ & $8,52 \mathrm{a}$ & $9,75 \mathrm{a}$ & $8,83 a$ & 26,14 & 0,62 & 2,43 \\
\hline CMS $\mathrm{g} / \mathrm{kg}^{0.75}$ & $93,15 \mathrm{a}$ & $91,58 \mathrm{a}$ & $83,69 a$ & $93,63 a$ & $85,52 \mathrm{a}$ & 28,14 & 0,74 & 0,02 \\
\hline CMS \% PV & $1,93 \mathrm{a}$ & $1,99 \mathrm{a}$ & $1,79 \mathrm{a}$ & $1,99 \mathrm{a}$ & $1,82 \mathrm{a}$ & 29,0 & 0,76 & 0,55 \\
\hline $\mathrm{CPB}, \mathrm{kg} / \mathrm{dia}$ & $1,27 \mathrm{a}$ & $1,55 \mathrm{a}$ & $1,66 \mathrm{a}$ & $1,60 \mathrm{a}$ & $1,38 \mathrm{a}$ & 24,44 & 0,21 & 0,36 \\
\hline CED, Mcal/dia & $37,11 \mathrm{a}$ & $36,81 \mathrm{a}$ & $33,50 \mathrm{a}$ & $37,65 \mathrm{a}$ & $34,09 \mathrm{a}$ & 26,37 & 0,68 & 9,46 \\
\hline PCQ, kg & $302,63 a$ & $277,50 \mathrm{a}$ & $283,14 \mathrm{a}$ & $286,50 a$ & $290,36 a$ & 8,43 & 0,49 & 24,34 \\
\hline $\mathrm{RCQ}, \%$ & $58,67 \mathrm{a}$ & $58,76 \mathrm{a}$ & $58,77 \mathrm{a}$ & $58,9 \mathrm{a}$ & $58,6 \mathrm{a}$ & 4,04 & 2,37 & 0,99 \\
\hline
\end{tabular}

Médias seguidas por letras distintas na mesma linha diferem entre si pelo teste de Duncan $(\mathrm{P}<0.05)$.

Peso vivo final (PVF), ganho total (GT), ganho médio diário (GMD), eficiência alimentar (EF), consumo individual de MS (CIMS), CMS em função do peso metabólico (CMS g/ $\mathrm{kg}^{0.75}$ ), consumo de MS em \% do peso vivo (CMS \% $\mathrm{PV})$, consumo de proteína bruta (CPB), consumo de energia digestível (CED), peso da carcaça quente (PCQ), rendimento da carcaça quente (RCQ).

SIL - silagem de milho e concentrado (grão de milho, farelo de soja, casca de soja, ureia e suplemento mineral) na proporção de 25:75 volumoso:concentrado (base na MS); PEL - dieta exclusiva de concentrado em pellets; GRN dieta com $85 \%$ de milho grão inteiro e $15 \%$ de concentrado em pellets.

Resultados contrários foram relatados por May et al. (2011), que testaram duas concentrações de grãos úmidos de destilaria $(15$ e $30 \%)$ e três concentrações de feno de alfafa $(7,5 ; 10$ e $12,5 \%)$ em comparação com dieta controle contendo $0 \%$ de grãos úmidos de destilaria e $10 \%$ de feno de alfafa. Não foram encontradas diferenças estatísticas entre os tratamentos $(\mathrm{P}>0,15)$ para PVF, GMD e PV ${ }^{0,75}$. De acordo com os autores, o CMS tendeu a aumentar linearmente à medida que a concentração de feno de alfafa aumentou na dieta, no entanto causou diminuição da EF $(\mathrm{P}<0,04)$.

Os resultados de CIMS e CMS (\%PV) foram semelhantes tanto para as dietas quanto para os grupos genéticos $(\mathrm{P}>0,05)$ (Tab. 2). Nkrumah et al. (2006), ao trabalharem com 27 novilhos, filhos de touros Angus ou Charolês, alimentados com dietas de alto concentrado, à base de milho no primeiro ano e à base de cevada e aveia no segundo ano, relataram valores mais elevados para CMS do que o presente estudo, de 11,62, 11,07 e 9,62kg/dia, sendo testadas várias ingestões residuais de matéria seca de dietas de alto concentrado. Não foram observadas diferenças no CMS $\left(\mathrm{g} / \mathrm{kg}^{0},{ }^{75}\right)$ em ambos os grupos genéticos e nas dietas utilizadas no presente estudo $(\mathrm{P}>0,05)$.

Restle et al. (2002), ao trabalharem com animais Braford, com alimentação com $55 \%$ de volumoso e $45 \%$ de concentrado, terminados com 14 meses de idade, relataram valores de 2,54\% de ingestão em função do PV. Resultados semelhantes foram relatados por Clímaco et al. (2011), em que os animais cruzados $1 / 2$ Bonsmara X 1/2 Nelore (B1) e 1/2 Bonsmara + 1/4 Red Angus $+1 / 4$ Nelore (B2), ingeriram de 2,2\% do PV, diferindo puro Tabapuã, que obteve $1,9 \%$ do PV. Também os mesmos autores relataram valores de $\mathrm{IMS}^{0,75}$ de $102 \mathrm{~g} / \mathrm{kg}^{0,75}$ para B1 e B2 e de $96 \mathrm{~g} / \mathrm{kg}^{0,75}$ para Bonsmara, sendo esses valores mais elevados do que os encontrados no presente estudo, e ainda, de $87 \mathrm{~g} / \mathrm{kg}^{0,}{ }^{75}$ para os animais Tabapuã, sendo este último valor próximo ao que foi encontrado para os cruzados do presente estudo. Ferrel e Jenkins (1998) relataram resultados mais baixos de consumo para animais Brahman alimentados com ingestão controlada ou ad libitum. Os animais iniciaram o experimento com $313 \mathrm{~kg}$ e obtiveram CMS de $7,35 \mathrm{~kg}$ MS/dia e ingestão de $86,2 \mathrm{~g} / \mathrm{kg}^{0,75}$ para os animais alimentados ad libitum. Esses valores foram semelhantes aos obtidos pelos cruzados Brahman do presente estudo. Com esses resultados, os animais Brahman do estudo de Ferrel e Jenkins (1998) obtiveram ganho total de $177,8 \mathrm{~kg}$, com ganhos de $1,462 \mathrm{~kg} / \mathrm{dia}$, em um período de confinamento de 140 dias, inferiores aos resultados deste estudo.

Os valores de CMS $\mathrm{g} / \mathrm{kg}^{0.75}$ (Tab. 2) foram inferiores aos previstos pelo NRC (1996), que é 
de $102 \mathrm{~g} / \mathrm{kg}^{0.75}$, e aos valores relatados por Clímaco et al. (2011) para o B1(1/2 Bosmara + 1/2 Nelore) e B2 (1/2 Bosmara + 1/4 Red Angus + 1/4 Nelore) mestiços. Apesar disso, valores semelhantes ao presente estudo foram relatados por Brondani et al. (2004), de $91,4 \mathrm{~g} / \mathrm{kg}^{0.75}$ para animais Angus e de $86,4 \mathrm{~g} / \mathrm{kg}^{0.75}$ para animais Hereford com a diferença entre os grupos genéticos $(\mathrm{P}<0,04)$. Nesse trabalho, NEL e NBR apresentaram valores de CMS $\mathrm{g} / \mathrm{kg}^{0.75}$, sem diferença estatística $(\mathrm{P}>0,05)(\mathrm{Tab} .2)$.

No trabalho de pesquisa de Ítavo et al. (2002), em que foram avaliados novilhos Nelore sob dietas com níveis crescentes de concentrado (20, 40,60 e $8 \%$ na MS) e dois teores de proteína bruta $(15$ e 18\%), foram relatados valores de consumo de 83,81 e $1,92 \%$ para nível de concentrado de $60 \%$ e de $84,15 \mathrm{~g} / \mathrm{kg}^{0.75}$ e $1,93 \%$ PV para concentrado a $80 \%$ na fase de terminação. Esses resultados foram semelhantes para as dietas SIL, PEL e GRN nesse experimento $(\mathrm{P}>0,05)$.

Nenhuma diferença significativa foi observada para CPB $(\mathrm{P}>0,05)$ entre as dietas e os grupos genéticos. Brondani et al. (2004) também não observaram efeito do grupo genético sobre essa variável. Ítavo et al. (2002) relataram uma diminuição linear no CMS e CPB conforme o aumento do nível de concentrado na dieta. Os resultados de CPB foram menores que os do presente estudo para dietas com $60 \%$ ou $80 \%$ de concentrado $(1,17$ e $1,10 \mathrm{~kg}$ de $\mathrm{PB} / \mathrm{dia}$, respectivamente).

As dietas e os grupos genéticos não influenciaram o consumo de energia digestível (CED) $(\mathrm{P}>0,05)$, com valores superiores aos obtidos por Brondani et al. (2004). Ao trabalharem com dietas contendo 12 ou $32 \%$ de concentrado, esses autores observaram que a diferença entre o CMS (\%PV) aumentou em 7\% para dieta com maior nível de concentrado.

As dietas e os grupos genéticos não influenciaram o PCQ e o RCQ (P>0,05). Resultados semelhantes foram observados por Ibrahim et al. (2008), que não encontraram diferenças para PCQ entre os grupos Waguli (Wagyu x Tuli) e Brahman e entre as duas dietas experimentais $(86 \%$ e $94 \%$ de concentrado na dieta) $(\mathrm{P}>0,05)$. Para RCQ, é possível observar que os animais NBR obtiveram um GT menor $(\mathrm{P}<0,05)$ em relação aos animais NEL (Tab. 2), o que mostra que o efeito genético aditivo do cruzamento NBR, a fim de aumentar o rendimento de carcaça e o ganho total, não foi suficiente para proporcionar valores maiores para essa variável. Resultados semelhantes foram relatados por Ribeiro et al. (2008), com médias de $51,1 \%$ para os animais Nelore e $50,8 \%$ para Nelore x Brahman (P>0,05). Clímaco et al. (2011) observaram melhores resultados para o grupo genético Tabapuã em relação ao Bonsmara e aos cruzamentos B1( $1 / 2$ Bosmara $+1 / 2$ Nelore $)$ e B2 (1/2 Bosmara + 1/4 Red Angus + 1/4 Nelore) para RCQ, com valor de 56,2\%. Porém, no presente estudo, o RCQ entre os grupos genéticos foi semelhante devido a uma ingestão de energia e proteína semelhante estatisticamente e, portanto, não se esperaria um aumento nessa variável.

A avaliação econômica mostrou que o confinamento foi viável economicamente para ambos os grupos genéticos e as dietas, apresentando valores de margem líquida positivos, ou seja, as receitas totais obtidas para cada dieta foram capazes de pagar os custos operacionais totais (COT) (Tab. 3). Resultados semelhantes foram descritos por Pacheco et al. (2006), que, ao avaliarem o confinamento de novilhos precoce e superprecoce dos grupos genéticos 5/8Charolês (CH) 3/8Nelore (NE) e $5 / 8 \mathrm{NE} \times 3 / 8 \mathrm{CH}$, respectivamente, observaram que ambos os grupos foram viáveis economicamente, pois a receita líquida total foi capaz de pagar o COT e possibilitou lucratividades total e mensal positivas. No entanto, animais $5 / 8 \mathrm{CH} \times 3 / 8 \mathrm{NE}$ proporcionaram maior receita líquida total e lucratividades total e mensal mais altas que os $5 / 8 \mathrm{NE} 3 / 8 \mathrm{CH}$.

Ao avaliarem dietas de alto grão adicionadas em diferentes porcentagens ( 0 a $15 \%)$ de volumoso (silagem de feno ou sob a forma de pellet), em confinamento de novilhos da raça Holandesa, Traxler et al. (1995) observaram também que os animais que consumiram milho inteiro e pellets apresentaram maior EF $(\mathrm{P}<0,01)$ e foram mais lucrativos em comparação aos outros tratamentos. Apesar de os resultados para a dieta GRN em relação à EF corroborarem esta afirmação, no presente estudo a dieta GRN foi estatisticamente igual tanto com SIL quanto com PEL para as margens líquidas e receitas observadas ao final do experimento. 
Tabela 3. Indicadores econômicos de bovinos Nelore e Nelore x Brahman sob diferentes dietas em confinamento

\begin{tabular}{|c|c|c|c|c|c|c|c|c|}
\hline & \multicolumn{3}{|c|}{ Dietas } & \multicolumn{2}{|c|}{ Grupo genético } & \multirow{2}{*}{$\mathrm{CV}$} & \multirow{2}{*}{$\begin{array}{c}\text { Valo } \\
\text { r-P }\end{array}$} & \multirow{2}{*}{ Erro } \\
\hline & SIL & PEL & GRN & NEL & NBR & & & \\
\hline COT, R\$ & $334,90 a$ & $397,58 \mathrm{a}$ & $385,16 a$ & $389,08 \mathrm{a}$ & $352,58^{\mathrm{a}}$ & 26,05 & 0,54 & 96,62 \\
\hline $\mathrm{COT}, \mathrm{R} \$ / \mathrm{kg}$ de carcaça & $2,87 \mathrm{~b}$ & $4,64 \mathrm{a}$ & $3,91 \mathrm{ab}$ & $3,78 \mathrm{a}$ & $3,75^{\mathrm{a}}$ & 32,80 & 0,12 & 1,24 \\
\hline Custo da dieta, $\mathrm{R} \$ / \mathrm{kg}$ & $0,22 \mathrm{c}$ & $0,35 b$ & $0,37 \mathrm{a}$ & $0,31 \mathrm{a}$ & $0,30 \mathrm{~b}$ & - & - & - \\
\hline Custo@produzida,R\$ & $43,17 b$ & $69,70 \mathrm{a}$ & $58,64 \mathrm{ab}$ & $56,74 \mathrm{a}$ & $56,34^{\mathrm{a}}$ & 32,78 & 0,12 & 18,54 \\
\hline $\mathrm{RT}, \mathrm{R} \$$ /cabeça & $596,71 \mathrm{a}$ & $438,21 b$ & $511,07 \mathrm{ab}$ & $547,84 \mathrm{a}$ & $490,23^{\mathrm{a}}$ & 15,52 & 0,03 & 80,53 \\
\hline ML, R $\$ /$ cabeça & $261,82 \mathrm{a}$ & $63,35 b$ & $125,92 \mathrm{ab}$ & $173,21 \mathrm{a}$ & $137,64^{\mathrm{a}}$ & 88,29 & 0,15 & 137,22 \\
\hline $\mathrm{ML}, \mathrm{R} \$ / \mathrm{kg}$ de carcaça & $2,12 \mathrm{a}$ & $0,35 \mathrm{~b}$ & $1,09 \mathrm{ab}$ & $1,24 \mathrm{a}$ & $1,21^{\mathrm{a}}$ & 100,42 & 0,12 & 1,24 \\
\hline
\end{tabular}

Médias seguidas por letras distintas na mesma linha diferem entre si pelo teste de Tukey $(\mathrm{P}<0,05)$.

Custo operacional total (COT), receita total (RT), margem líquida (ML).

SIL - silagem de milho e concentrado (grão de milho, farelo de soja, casca de soja, ureia e suplemento mineral) na proporção de 25:75 volumoso: concentrado (base na MS); PEL - dieta exclusiva de concentrado em pellets; GRN dieta com $85 \%$ de milho grão inteiro e $15 \%$ de concentrado em pellets.

Ao se avaliarem as três dietas, pode-se notar que os custos operacionais totais foram semelhantes, diferindo somente quando o COT é apresentado em função de $\mathrm{kg} /$ carcaça produzida; nesse caso, a dieta PE é a que apresenta maior $\mathrm{COT} / \mathrm{kg}$, diferindo estatisticamente da dieta SIL $(\mathrm{P}<0,05)$ e ambas semelhantes à GRN (P>0,05). Verificase que este maior custo é em função do consumo, do RCQ e do GMD (P>0,05), em que as dietas PEL e GRN, apesar de mesmo consumo e RCQ, obtiveram menores GMD $(\mathrm{P}<0,05)$, consequentemente gerando um GT menor $(\mathrm{P}<0,05)$, quando comparadas com a dieta SIL.

A RT foi maior para a dieta SIL em relação à PEL $(\mathrm{P}<0,05)$, e ambas foram semelhantes à GRN, sendo que esses valores verificados são em função das diferenças de GMD e EF. A maior margem líquida (lucro operacional) foi observada para SIL, seguida de GRN e PEL (Tab. 3), sendo que SIL apresentou resultado estatisticamente maior que PEL $(\mathrm{P}<0,05)$. Assim, a SIL apresentou melhor resultado econômico em razão da margem líquida, em comparação à PEL $(\mathrm{P}<0,05)$, e, além disso, foi a dieta que apresentou o maior GMD durante todo o período de confinamento. A margem líquida da GRN foi estatisticamente semelhante à SIL $(\mathrm{P}>0,05)$, o que é explicado pela semelhante EF de ambas $(\mathrm{P}>0,05)$, apesar de menor GMD da dieta GRN $(\mathrm{P}<0,05)$.

Diferentemente do presente experimento, Missio et al. (2009) observaram redução de receita com o aumento de concentrado, chegando a ser negativa $(\mathrm{R} \$-4,13)$ com concentrado na faixa de $79 \%$ da dieta, o que inviabilizou o confinamento. Esses autores relacionaram o resultado aos altos custos dos insumos do concentrado.

Ao se avaliar o custo da arroba produzida, é possível observar que o menor custo, entre as três dietas, foi para SIL, com $\mathrm{R} \$ 43,17$, sendo similar à GRN $(\mathrm{P}>0,05)$. Todavia, o maior custo da@ @roduzida foi para PEL, apesar de o custo da dieta $(\mathrm{R} \$ / \mathrm{kg})$ não ser o maior entre as três, o que indica que, ao se avaliar somente o custo da dieta, não necessariamente se terá o melhor resultado econômico, sendo fundamental analisar em conjunto o desempenho (Tab. 2), para concluir sobre os motivos do maior COT. Deste modo, verifica-se que a dieta PEL obteve menor eficiência técnica e econômica em relação às demais, gerando, assim, um menor ganho de peso, apesar de consumos semelhantes, maior custo de kg de carcaça e menor margem líquida.

\section{CONCLUSÕES}

O cruzamento NBR não foi capaz de promover ganhos produtivos e econômicos adicionais em comparação ao grupo NEL, apresentando desempenhos semelhantes. Todas as dietas foram capazes de pagar os custos e remunerar o produtor, no entanto a dieta SIL proporcionou maior ganho de peso e melhor margem líquida. 


\section{REFERÊNCIAS}

ASSOCIATION of Official Analytical Chemists - AOAC. Official methods of analysis. 15ed. Virginia. 359p. 1990.

BENTON, J.R.; ERICKSON, G.E.; KLOPFENSTEIN, T.J. et al. Effects of roughage source and level in finishing diets containing wet distillers grains on feedlot performance and economics. J. Anim. Sci., v.85, p.76, 2007. (Abstr.)

BRASIL. MAPA - Ministério da Agricultura, Pecuária e Abastecimento, Regulamento Técnico de Métodos de Insensibilização para o Abate Humanitário de Animais de Açougue. Instrução Normativa $n^{\circ} 3$, de 17 de janeiro de 2000.

BRONDANI, I.L.; SAMPAIO, A.A.M.; RESTLE, J. et al. Desempenho de bovinos jovens das raças Aberdeen Angus e Herefod, confinados e alimentados com dois níveis de energia. Rev. Bras. Zootec., v.33, p.2308-2317, 2004.

BUNGENSTAB, E.J., ALMEIDA, A.J. Avaliação de custos em confinamento de bovinos de corte no Brasil. In: REUNIÃO DA SOCIEDADE BRASILEIRA DE ZOOTECNIA, 38., Piracicaba. Anais ... Piracicaba: SBZ, 2001. p 1514-1516.

CAPPELE, E.R.; VALADARES FILHO, S.C.; SILVA, J.F.C. et al. Estimativas do valor energético a partir de características químicas e bromatológicas dos alimentos. Rev. Bras. Zootec., v.30, p.1837-1856, 2001.

CLÍMACO, $\quad$ S.M.; RIBEIRO, $\quad$ E.L.A.; MIZUBUTI, I.Y. et al. Performance and carcass traits of four genetic groups of beef cattle steers finished in feedlot. Rev. Bras. Zootec., v.40, p.1562-1567. 2011.

CORRÊA, E.S.; VIEIRA, A.; COSTA, F.P. et al. Sistema semi-intensivo de produção de carne de bovinos nelores no Centro-Oeste do Brasil. Embrapa/CNPGC: Campo Grande, 2000.

CUNDIFF, L.V.; SAZBO, F.; GREGORY, K.E. et al. Breed comparsions in the germoplasm evaluation program at MARC. In: BEEF IMPROVEMENT FEDERATION $25^{\text {th }}$ ANNIVERSARY CONFERENCE. Asheville. 1993. Disponível em: http://westnilevirus. okstate.edu/breeds/research/marccomp.pdf Acesso em: 06/12/2011.
FERREL, C.L.; JENKINS, T.G. Body composition and energy utilization by steers of diverse genotypes fed a high-concentrate diet during the finishing period: II. Angus, Boran, Brahman, Hereford, and Tuli sires. J. .Anim. Sci., v.76, p.647-657, 1998.

IBRAHIM, R.M.; GOLL, D.E.; MARCHELLO, J.A. et al. Effect of two dietary concentrate levels on tenderness, calpain and calpastatin activities and carcass merit in Waguli and Brahman steers. J. Anim. Sci., v.86, p.1426-1433, 2008.

ÍTAVO, L.C.V.; VALADARES FILHO, S.C.; SILVA, F.F., et al. Níveis de concentrado e proteína bruta na dieta de bovinos Nelore nas fases de recria e terminação: Consumo e Digestibilidade. Rev. Bras. Zootec., v.31, p.10331041, 2002.

KIPPERT JUNIOR, C.; RORATO, P.R.N.; LOPES, J.S. et al. Efeitos genéticos aditivos diretos e maternos e heterozigóticos sobre os desempenhos pré e pós-desmame em uma população multirracial Aberdeen Angus $\mathrm{x}$ Nelore. Rev. Bras. Zootec., v.37, p.1383-1391. 2008.

KREIKERMEIER, K.K.; HARMON， D.L.; BRANDT Jr., R.T. et al. Steam-rolled wheat diets for finishing cattle: effects of dietary roughage and feed intake on finishing steer performance and ruminal metabolism. J. Anim. Sci., v.68, p.2130-2141, 1990.

LOPES, M.A.; CARVALHO, F.M. Custo de produção de gado de corte. Lavras: UFLA, 2002. 47p.

LOPES, M.A.; MAGALHÃES, G.P. Análise da rentabilidade da terminação de bovinos de corte em condições de confinamento: um estudo de caso. Arq. Bras. Med. Vet. Zootec., v.57, p.374379, 2005.

MAY, M.L.; QUINN, M.J.; DILORENZO, N. et al. Effects of roughage concentration in steam-flaked corn-based diets containing wet distillers grains with soluble on feedlot cattle performance, carcass characteristics and in vitro fermentation. J. Anim. Sci., v.89, p.549-559, 2011. 
MISSIO, R.L.; BRONDANI, I.L.; FREITAS, L.S. et al. Desempenho e avaliação econômica da terminação de tourinhos em confinamento alimentados com diferentes níveis de concentrado na dieta. Rev. Bras. Zootec., v.38, p.1309-1316, 2009.

NKRUMAH, J.D.; OKINE, E.K.; MATHISON, G.W., et al. Relationship of feedlot feed efficiency, performance, and feeding behavior with metabolic rate, methane production, and energy partitioning in beef cattle. J. Anim. Sci., v.84, p.145-153, 2006.

NOGUEIRA, M.P. Gestão de custos e avaliação de resultados: agricultura e pecuária. Bebedouro: Scot Consultoria, 2004. 219 p.

NUTRIENT Requeriments of beef cattle. 7.ed. Washington: National Academy of Sciences, 1996. 242p

PACHECO, P.S.; RESTLE, J.; VAZ, F.N. et al. Avaliação econômica da terminação em confinamento de novilhos jovens e superjovens de diferentes grupos genéticos. Rev. Bras. Zootec., v.35, p.309-320, 2006.

RIBEIRO, E.L.A.; HERNANDEZ, J.A.; LOURENÇO, E.Z. et al. Desempenho e características da carcaça de diferentes grupos genéticos de novilhos. Rev. Bras. Zootec., v.37, p.1669-1673, 2008.

REIS, R.P. Fundamentos de economia aplicada. Lavras: UFLA/FAEPE, 2002. 95p.
RESTLE, J.; NEUMANN, M.; BRONDANI, I.L. et al. Manipulação da altura de corte da planta de milho (Zea mays, L.) para ensilagem visando a produção do novilho superprecoçe. Rev. Bras. Zootec., v.31, p.1235-1244, 2002.

STATISTICAL Analysis System - SAS. INSTITUTE. SAS/STAT User's guide. Version 6, 4.ed., v.1, Cary, 1990.

SMITH, A.M.; REID, J.T. Use of chromic oxide as an indicator of fecal output for the purpose of determining the intake of a pasture herbage by grazing cows. J. Dairy Sci., v.38, p.515-524, 1955.

TILLEY, J.M.A.; TERRY, R.A. A two stage technique for the in vitro digestion of forage crops. J. Br. Grassland Soc., v.18, p.104, 1963.

TRAXLER, M.J.; FOX, D.G.; PERRY, T.C. et $a l$. Influence of roughage and grain processing in high-concentrate diets on the performance of long-fed Holstein steers. J. Anim. Sci., v.73, p.1888-1900, 1995.

VAN SOEST, P.J., ROBERTSON, J.B., LEWIS, B.A. Methods for dietary fiber, neutral detergent, and nonstarch polysaccharides in relation to animal nutrition. J. Dairy Sci., v.74, p.35833597, 1991.

WILLIAMS, C.H.; DAVID, D.J.; ISMAA, O. The determination of chromic oxide in feces samples by atomic absorption spectrophometry. J. Agricult. Sci., v.59, p.381-385, 1962. 